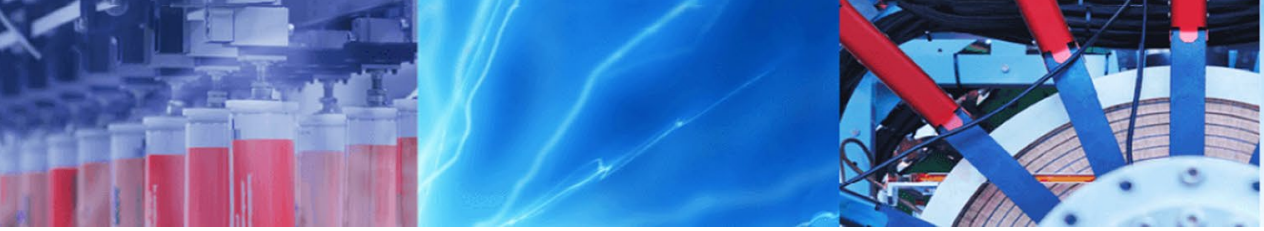

Research Article

\title{
Luminescent PVDF nanocomposite films and fibers encapsulated with $\mathrm{La}_{2} \mathrm{Hf}_{2} \mathrm{O}_{7}: \mathrm{Eu}^{3+}$ nanoparticles
}

\author{
Santosh K. Gupta ${ }^{1} \cdot$ Carlos Hernandez $^{2} \cdot$ Jose P. Zuniga $^{3} \cdot$ Karen Lozano $^{2} \cdot$ Yuanbing Mao ${ }^{4}$
}

Received: 25 January 2020 / Accepted: 2 March 2020 / Published online: 12 March 2020

(c) Springer Nature Switzerland AG 2020

\begin{abstract}
Luminescent films and fibers are in high demand in display devices and optoelectronics owing to their higher flexibility and mechanical strength compared to powder samples. Keeping that in mind, we have encapsulated $\mathrm{La}_{2} \mathrm{Hf}_{2} \mathrm{O}_{7}: \mathrm{Eu}^{3+}$ (LHOE) nanoparticles (NPs) synthesized by a molten salt method into polyvinylidene fluoride (PVDF) films and fibers. The LHOE-PVDF nanocomposite fibers were manufactured using a Forcespinning ${ }^{\circledR}$ technology while the counterpart films were fabricated by a drop-casting technique. Their photophysical properties were analyzed and compared. Specifically, the red emission with optimum lifetime, color coordinates, and branching ratio of the LHOE NPs can be reproduced even when incorporated inside the PVDF films and fibers. Our studies demonstrate that the LHOE-PVDF films have better emission output, excited-state lifetime, quantum yield, and color purity than the fibers. This is attributed to lower defect density and better surface texture of the LHOE-PVDF films compared to the fibers. Based on the Judd-Ofelt analysis, we have also elucidated that the Eu-O bond is more polarizable and has a higher degree of covalency in the LHOE-PVDF films compared to that in the fibers. The trend of the Judd-Ofelt parameters suggested that $\mathrm{Eu}^{3+}$ has a more asymmetric environment in the films compared to the fibers which impart higher red color purity to the former.
\end{abstract}

Keywords Pyrochlore PVDF · Europium · Nanocomposite · Luminescence

\section{Introduction}

Luminescent nanophosphors (LNPs) have been an epitome of scientific research owing to their excellent optical properties, tunable light emission, large stokes shift, etc. For the same reason, they have been getting significant attention for applications in solid-state lighting, scintillators, thermal sensor, pressure sensor, bioimaging, catalysis, biosensor, photodynamic therapy, fingerprint imaging, etc. [1-9]. Synthesis of LNPs plays a vital role in deciding its optimal usage and designated field [10]. This is because synthesis methods control the size, shape, defect density and final quality of LNPs [10-12]. Most of the reported LNPs have been synthesized in powder form using several methods such as sol-gel [13,14], combustion [9], polymeric precursor [15], molten salt synthesis $[12,16]$, hydrothermal [17], reverse micellar route [18], etc.

Based on earlier reports, $\mathrm{La}_{2} \mathrm{Hf}_{2} \mathrm{O}_{7}$ have been proposed as a good photo- and radio-luminescent host for lanthanide-doped phosphor materials. Some of the favorable properties include excellent dielectric properties due to its small number of defect densities, ability to accommodate large concentration of dopant both at $A$ and $B$ sites with a general formula of $A_{2} B_{2} \mathrm{O}_{7}$, wide bandgap, moderate phonon $E=745 \mathrm{~cm}^{-1}$ and high stopping power for $X$ - and $Y$-rays with $Z_{\mathrm{Hf}}=72$, and high density of $7.9 \mathrm{~g} / \mathrm{cm}^{3}$. [19-21] We have done extensive work on red-emitting $\mathrm{La}_{2} \mathrm{Hf}_{2} \mathrm{O}_{7}: \mathrm{Eu}^{3+}$ (LHOE) down-converting nanoparticle (NP)

$\triangle$ Santosh K. Gupta, santoshg@barc.gov.in; Yuanbing Mao, ymao17@iit.edu| ${ }^{1}$ Radiochemistry Division, Bhabha Atomic Research Centre, Trombay, Mumbai 400085, India. ${ }^{2}$ Department of Mechanical Engineering, University of Texas Rio Grande Valley, Edinburg, TX 78539, USA. ${ }^{3}$ Department of Chemistry, University of Texas Rio Grande Valley, Edinburg, TX 78539, USA. ${ }^{4}$ Department of Chemistry, Illinois Institute of Technology, 3105 South Dearborn Street, Chicago, IL 60616, USA. 
powders for several applications in the areas of phosphors, scintillators, thermographic phosphors, etc. [5, 7, 21].

When it comes to commercial applications, powdered nanophosphors present a variety of issues which have precluded their use. Powdered nanoparticles suffer from low thermal stability, low photostability, poor mechanical strength, tendency to aggregate, and are prone to water adsorption on their surfaces [22].For application as light emitting devices, selected phosphor powders are first mixed with glues, and then the mixture is used to cover blue emitting semiconducting GaN/InGaN chips [23]. Though, adding the glue presents challenges, the thickness of the emissive layer is hard to control, and as a result, the reproducibility of light emission varies [24]. This could be tackled by incorporating phosphor particles within a polymer matrix $[23,24]$. It is also reported that some of the oxide phosphor powders readily get converted into hydroxide on storage [25]. However, degradation could be reduced by incorporating particle powders into polymer matrixes. It is also reported that encapsulating LNPs into polymeric matrices leads to various favorable properties such as enhanced flexibility, improved photostability, lower tendency to aggregate, etc. Therefore, encapsulation of LNPs in polymers could increase commercial applications [26-28].

The selection of polymer host is also critical. The polymer should be thermally/chemically stable, not absorb visible light, have high optical transparency, possess optimum refractive index, etc. [26]. Polyvinylidenefluoride (PVDF, $\mathrm{C}_{2} \mathrm{H}_{2} \mathrm{~F}_{2}$ ) is a semi-crystalline polymer that presents several advantages such as ease of synthesis, optimum mechanical strength, excellent thermal and chemical stability, etc. [8, 23, 29].

The amalgam of LHOE nanoparticles and PVDF which can be termed as the nanocomposite utilizes the positive aspects of the polymer such as lightweight and flexibility with the desirable properties of LHOE NPs such as narrow emission, high color purity, low phonon energy, and higher excited state lifetime. This synergy could impart such nanocomposite with remarkable capability for used in optoelectronics, solar cell, display panels, etc. $[27,30]$. In this study, we propose to develop LHOE-PVDF nanocomposites with distinct morphologies of fibers and films, to analyze and compare their luminescence properties. Luminescent nanocomposite fibers are expected to be more suitable for optical thermometry and optical sensor applications because they exhibit negligible interference from electromagnetic noises and are capable for localized measurements [31, 32]. 1D micro-fibers have recently gained lots of interest among the scientific community owing to their superior properties and applications in gas sensing, optoelectronics, photonics, optical pressure sensing, etc. [8, 31, 32]. Antoniadau et al. have designed up-converting nanocomposite fibers which are expected to have improved imaging applications [33].

As far as the film is concerned, assembling oxide phosphor powders into polymeric thin films has several advantages such as a more close-packed structure with less void, improved stability in high vacuum, and withstanding the high flux of electron bombardment, which make them highly suitable for flat display panels [34-36]. It has also been reported that films based on oxide phosphors display high image resolution and a strong tendency for adhesion to various substrates [37].

In this work, we used the Forcespinning ${ }^{\circledR}$ technology [38-40] to fabricate the nanocomposite fibers. Forcespinning presents several advantages over electrospinning, for example, no voltage is needed and the output is orders of magnitude higher [41]. To facilitate the commercial viability of red-emitting LHOE nano-phosphor, we have used these as reinforcements for a PVDF matrix. We used a drop-casting technique to fabricate the nanocomposite film. The developed LHOE reinforced PVDF nanocomposite fibers and films have been subjected to intense optical measurements and Judd-Ofelt analysis.

\section{Experimental}

\subsection{Synthesis}

$\mathrm{N}, \mathrm{N}$-Dimethylacetamide (DMA, $\geq 99 \%)$, acetone ( $\geq 99.5 \%)$, PVDF (average molecular weight of 534,000 ) were purchased from Sigma-Aldrich USA. The LHOE NPs were synthesized by a molten salt method as reported in our previous publications $[5-7,10] .1 .0 \mathrm{wt} \%$ of LHOE NPs and $22 \mathrm{wt} \%$ of PVDF were mixed in a $77 \mathrm{wt} \%$ solvent (50:50 weight percentage ratio of acetone: DMA) by stirring with a magnet bead for $2 \mathrm{~h}$. The LHOE-PVDF nanocomposite films were fabricated through a drop-casting technique by dropping $1 \mathrm{~mL}$ of the solution on a planar surface of a watch glass. The LHOE-PVDF nanocomposite fibers were produced via a Forcespinning ${ }^{\circledR}$ technique. A spinneret rotates to create a centrifugal force that spits and evaporates the polymer solution forming then fibers. A 30 -gauge needle was used to inject $2 \mathrm{~mL}$ of the polymer solution into the spinneret. The spinneret ran at a speed of $7000 \mathrm{rpm}$ for $8 \mathrm{~min}$. The fibers were gathered from the $1 " \times 1$ " aluminum collector square [42].

\subsection{Instrumentation}

Pristine LHOE NPs were characterized systematically using $X$-ray diffraction (XRD), Raman spectroscopy, and Scanning electron microscopy (SEM) for phase purity, structure, particle size, and morphology.XRD was conducted using 
a Bruker ${ }^{\top m} \mathrm{D} 8$ Advance powder diffractometer using a copper source with $\mathrm{Ka}_{1}$ radiation of $(\lambda=0.15406 \mathrm{~nm}, 40 \mathrm{kV}$, $40 \mathrm{~mA}$ ) and a scanning range from $10^{\circ}-90^{\circ}$ with a step size/rate of $\left(0.04^{\circ}\right.$, at $\left.2^{\circ} \mathrm{min}^{-1}\right)$. Furthermore, to determine the proper phase formation of the LHOE NPs, Raman spectroscopy was utilized with a Bruker Senterra system equipped with a $785 \mathrm{~nm}$ helium-neon laser with a spatial resolution of $2 \mathrm{~mm}$. In addition, to test the morphology and size of the synthesized LHOE NPs and the LHOE-PVDF nanocomposites, SEM was used. The Carl Zeiss sigma VP field emission SEM was operated at ( $1.0 \mathrm{kV} \& 2.0 \mathrm{kV})$. To accurately quantify the optical performance, excitation, emission, and lifetime, photoluminescence tests were carried out using an FLS 980 Edinburgh instrument equipped (resolution of $0.05 \mathrm{~nm}$ ) with a Xenon lamp capable of switching from continuous wave to pulse with a frequency range of $1-100 \mathrm{~Hz}$. The pulse width of excitation source is 1-2 microseconds. Every Edinburgh Instruments fluorescence spectrometer is shipped with a factory generated emission correction factor for each emission lightpath (detector and grating combination) in the spectrometer $[43,44]$. The details about how actually correction file is generated are given in reference 43 and 44 . Instrument has inbuilt correction software which take care of emission and excitations corrections w.r.t to source, monochromator and detector. This is done so that source emits constant photon output at all wavelength, monochromator allows photon of all wavelengths with equal efficiency and detector detects photon of all wavelength with equal efficiency. Source is in perpendicular configuration w.r.t to detector to avoid interference from more intense incident light beam. Yellow filter is used while recording the emission spectra to take care of higher order reflections.

\section{Results and discussion}

\subsection{Characterization of the LHOE NPs}

The as-prepared LHOE NPs were subjected to quality check before being encapsulated into the PVDF matrix. LHOE has an $\mathrm{A}_{2} \mathrm{~B}_{2} \mathrm{O}_{7}$ composition which could exist in either pyrochlore (OPP) or defect fluorite (DFP) structure due to their very similar cubic nature. The OPP structure has a space group of $(\mathrm{Fd} 3 \mathrm{~m})$ while the DFP structure belongs to the (Fm3m) group [45]. The powder XRD pattern of the LHOE NPs (Fig. 1a) demonstrates welldefined sharp peaks reflecting their crystalline nature. The reflection pattern matches completely with the DFP structure. The weaker OPP super lattice reflections are difficult to observe with weak Cu-Ka X-ray source and in most cases Synchroton based XRD is needed to observe the same. However, the XRD patterns show high purity of MSS LHOE NPs and no impurity of $\mathrm{La}_{2} \mathrm{O}_{3}, \mathrm{Eu}_{2} \mathrm{O}_{3}$, and $\mathrm{HfO}_{2}$ could be seen.

To differentiate the OPP and DFP structures, Raman spectroscopy has been utilized due to its high sensitivity to metal-oxygen(M-O) vibrations. The DFP structure shows only one $\mathrm{M}-\mathrm{O}$ vibrational mode under Raman spectroscopy that has been attributed to the $F_{2 g}$ mode. The seven $\mathrm{O}^{2-}$ ions being randomly distributed across the eight anionic sites cause a high degree of disorder in the crystal lattice allowing for only one vibrational mode of the DFP structure [46]. On the other hand, the pyrochlore structure shows a total of six vibrational modes located at $302 \mathrm{~cm}^{-1}, 320 \mathrm{~cm}^{-1}, 400 \mathrm{~cm}^{-1}$, $506 \mathrm{~cm}^{-1}, 520 \mathrm{~cm}^{-1}$, and $610 \mathrm{~cm}^{-1}$ correspondings to the $F_{2 g^{\prime}} \mathrm{E}_{g^{\prime}} F_{2 g^{\prime}} A_{1 g^{\prime}} F_{2 g^{\prime}}$ and $F_{2 g}$ vibrational mode respectively [47]. The Raman spectrum of the our LHOE NPs (Fig. $1 \mathrm{~b}$ ) as well showed a total of six activate vibrational modes of the OPP structure. The small peak of approximately $\sim 702 \mathrm{~cm}^{-1}$ has been attributed to a Hf-O octahedral distortion in the lattice.

Based on SEM we could found that the LHOE NPs have a spherical shape with a size ranging between $20-70 \mathrm{~nm}$ and low degree of aggregation exists between the particles (Fig. 1C). Several parameters affect the morphology and size of NPs synthesized by the molten salt method. In our previous investigation, we demonstrated that the level of $\mathrm{OH}^{-}$concentration during the synthesis of the precursor affects the size of the synthesized NPs without manipulating the molten salt synthesis time and temperature [7]. For the LHOE system, as the concentration of $\mathrm{OH}^{-}$increased, the size of the particles increases proportionally [7]. In the present study, the LHOE NPs were synthesized using a $10 \% \mathrm{NH}_{4} \mathrm{OH}$ aqueous solution, which agrees with the correlated size revealed by the SEM image analysis, Fig. 1c.

\subsection{SEM of the LHOE-PVDF films and fibers}

The two sets of SEM images shown in Fig. 2 are entirely different in terms of their surface texture and orientation. The SEM images of the LHOE-PVDF nanocomposite films (Fig. 2a, b) show an encapsulated spherical morphology with a variety of sizes. On the other hand, the SEM images of the LHOE-PVDF fibers (Fig. 2c, d) depict fibers of several micrometers in lengths with a high degree of entanglement. The surface of the nanocomposite microfibers appeared quite rough and most of the individual fibers have a non-uniform cross-section. The digital photographs of the LHOE-PVDF films and fibers (Fig. 2e, $f$ ) also indicate the formation of films and fibers from the LHOE-PVDF nanocomposite system. 

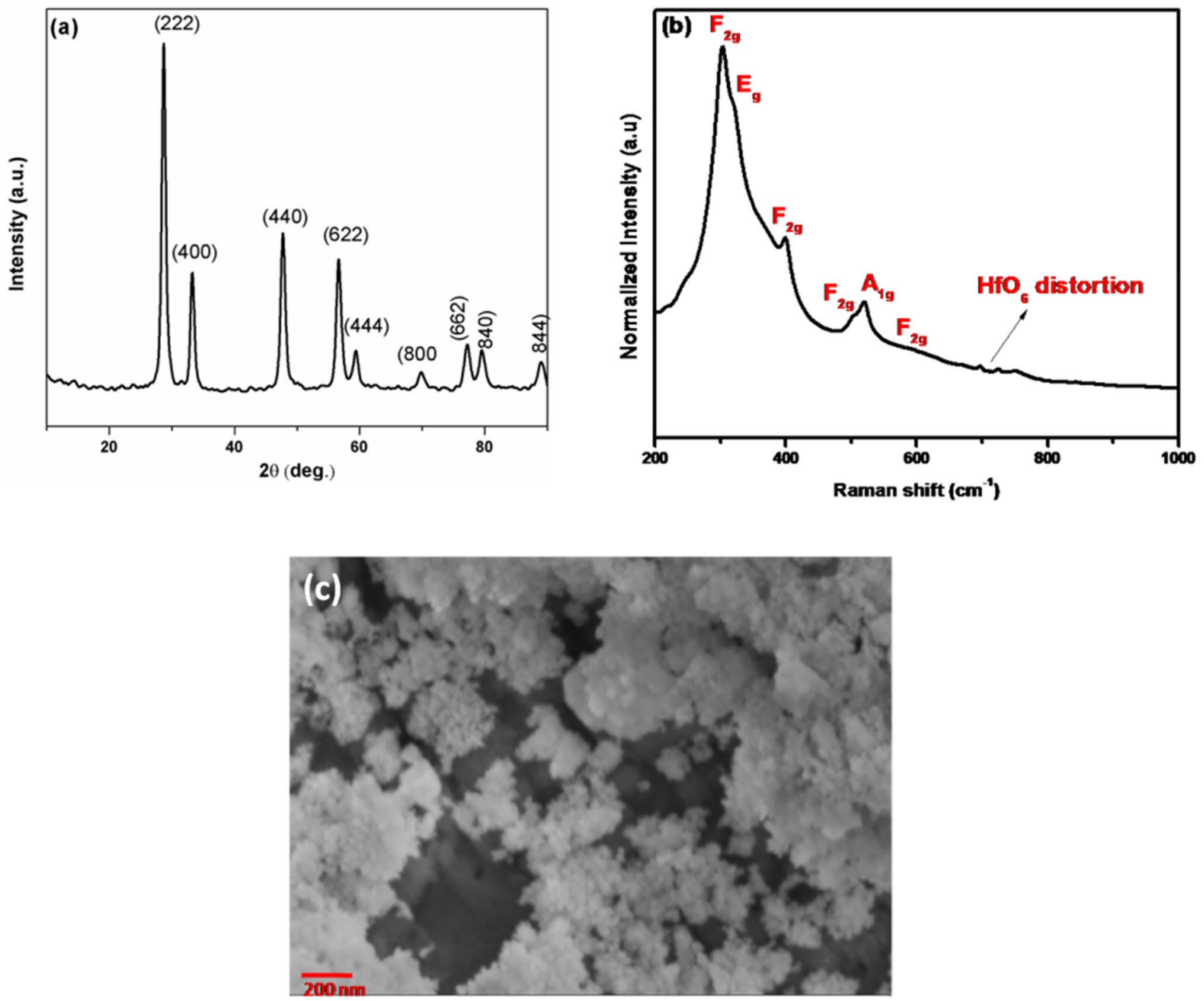

Fig. 1 a XRD pattern, b Raman spectrum, and c SEM image of the LHOE NPs synthesized by a molten salt method at $650^{\circ} \mathrm{C}$

\subsection{Excitation and emission spectra of the LHOE-PVDF films and fibers}

The excitation spectrum (Fig. 3a) of LHOE-PVDF nanocomposite film and fiber consists of dual features: a broad band (B1) in the range of $240-300 \mathrm{~nm}$ peaking around $254 \mathrm{~nm}$ with several fine peaks (B2) spanning within the $350-500 \mathrm{~nm}$ wavelength range. The broadband is attributed to $\mathrm{O}^{2-} \rightarrow \mathrm{Eu}^{3+}$ charge transfer whereas the fine structures appear due to intra $f-f$ transition of trivalent europium ion. The intensity of the allowed B1 peak is much higher from the films compared to the fibers. The known $\mathrm{f}$ - $f$ bands around $395 \mathrm{~nm}$ and $465 \mathrm{~nm}$ due to ${ }^{7} \mathrm{~F}_{0} \rightarrow{ }^{5} \mathrm{~L}_{6}$ and ${ }^{7} \mathrm{~F}_{0} \rightarrow{ }^{5} \mathrm{D}_{2}$ transitions are more prevalent from the fibers compared to the films. These results show that the films are better candidate for UV excitation while the fibers seem to be a better option when using near UV or blue excitation.

As the excitation peaks at $395 \mathrm{~nm}$ and $465 \mathrm{~nm}$ are almost absent in the excitation spectra of the LHOEPVDF films (Fig. 3a, bottom), the emission spectra of the films and fibers were conducted using $254 \mathrm{~nm}$ excitation (Fig. 3b). The emission spectra consist of features peculiar of trivalent europium ion: ${ }^{5} D_{0} \rightarrow{ }^{7} F_{1}$ magnetic dipole transition (MDT) at $590 \mathrm{~nm},{ }^{5} \mathrm{D}_{0} \rightarrow{ }^{7} \mathrm{~F}_{2}$ hypersensitive electric dipole transition (EDT) at $615 \mathrm{~nm}$, and the ${ }^{5} \mathrm{D}_{0} \rightarrow{ }^{7} \mathrm{~F}_{0}$, ${ }^{5} D_{0} \rightarrow{ }^{7} F_{3}$ and ${ }^{5} D_{0} \rightarrow{ }^{7} F_{4}$ transitions at around 577,653 and $712 \mathrm{~nm}$. Along with these transitions, the fiber emission spectra also displayed some emission from the ${ }^{5} D_{1}$ level which is usually not seen unless phonon energy in the system is low [15]. Typically, the origin of the MDT indicates the presence of inversion symmetry around $\mathrm{Eu}^{3+}$ ions. 

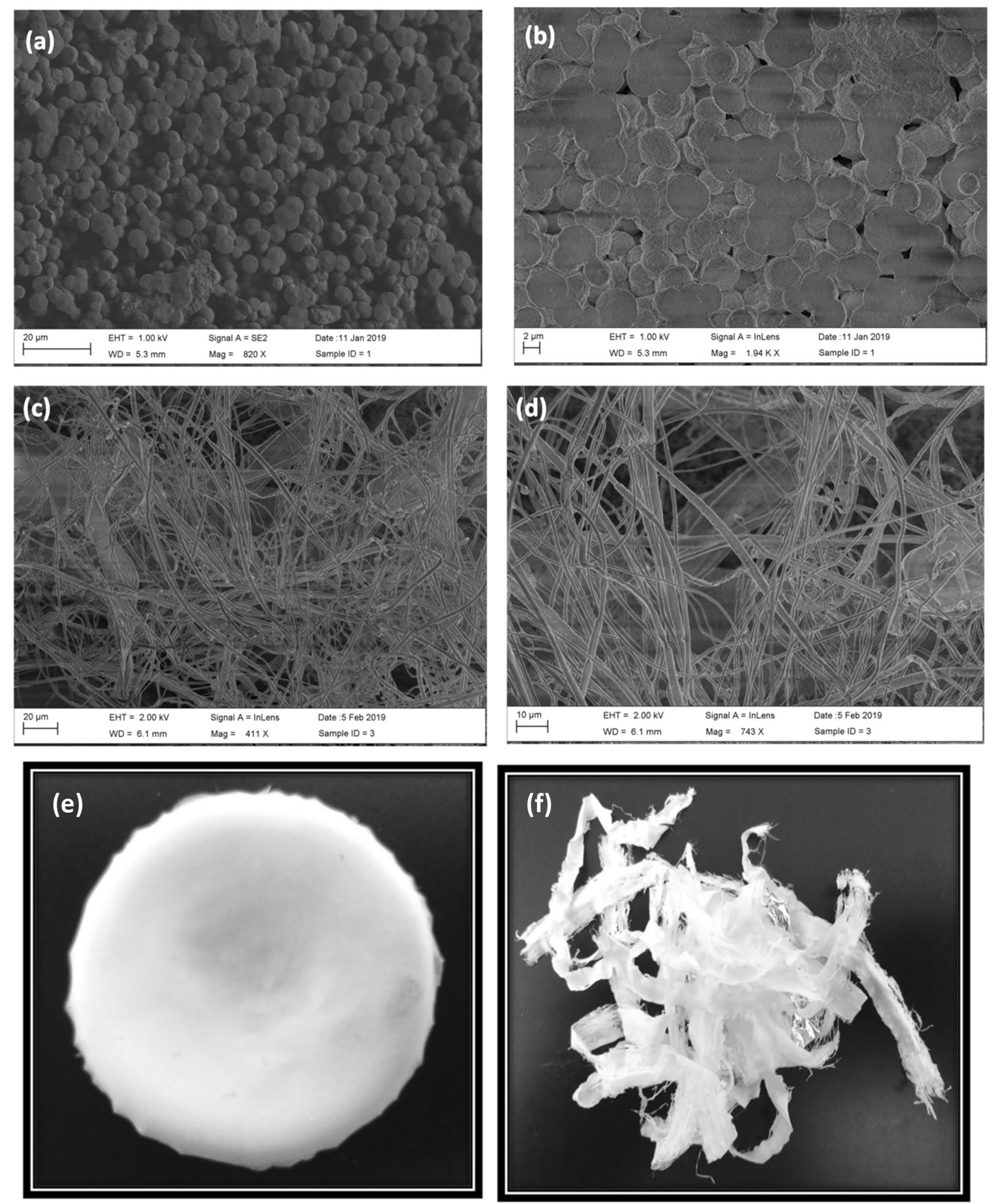

Fig. 2 SEM images of the LHOE-PVDF $\mathbf{a}, \mathbf{b}$ films and $\mathbf{c}, \mathbf{d}$ fibers. Digital images of the LHOE-PVDF nanocomposite $\mathbf{e}$ films and $\mathbf{f}$ fibers 

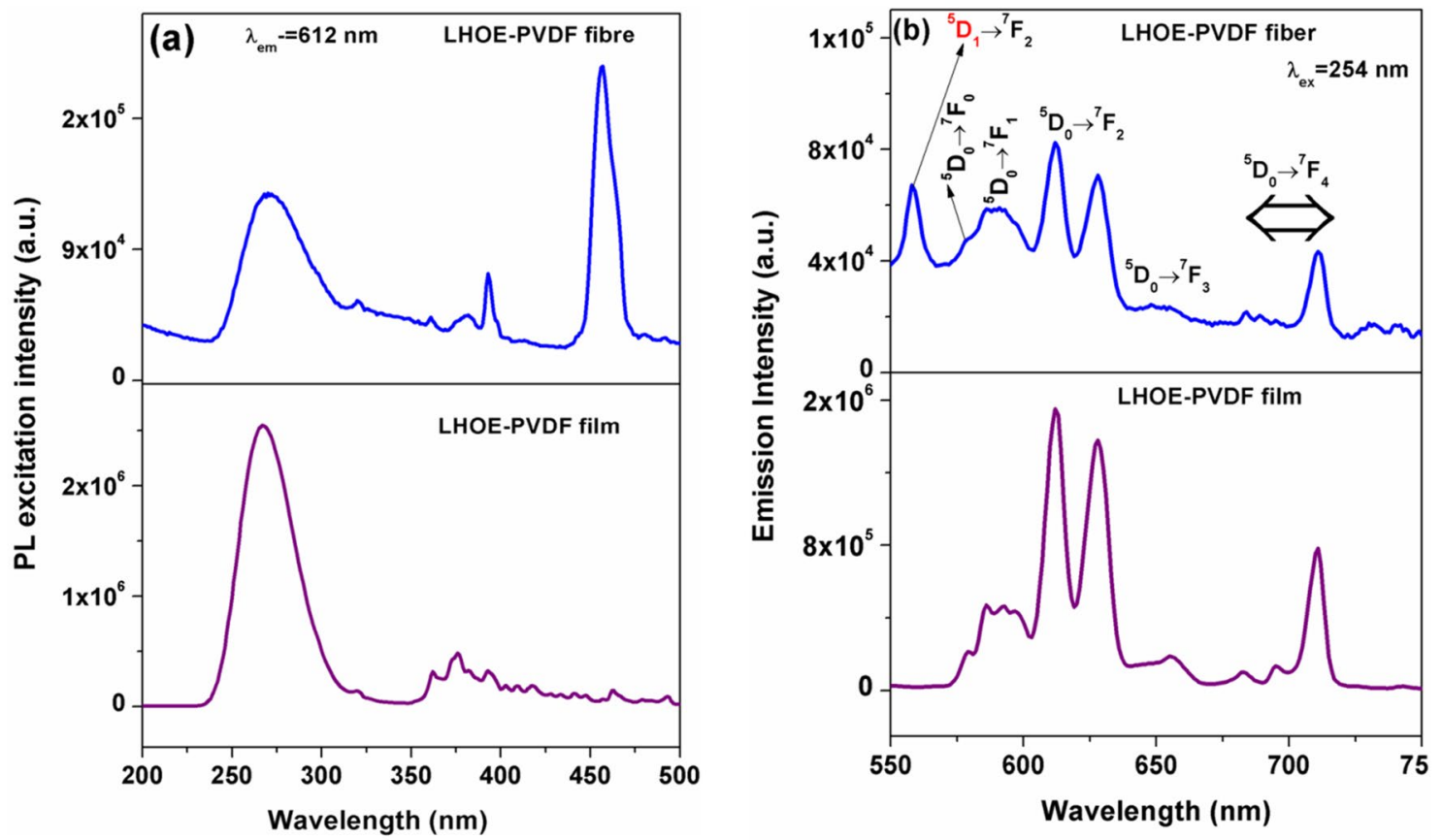

Fig. 3 a Excitation and $\mathbf{b}$ emission spectra of the LHOE-PVDF fibers (top) and films (bottom)

On the other hand, the EDT appears when $\mathrm{Eu}^{3+}$ ions are localized in a low symmetry environment. The fact that the intensity of EDT is higher than that of MDT in both samples suggests that $\mathrm{Eu}^{3+}$ ions lack inversion symmetry and the local symmetry around them is quite low, which is expected due to the combined field of $\mathrm{La}_{2} \mathrm{Hf}_{2} \mathrm{O}_{7}$ pyrochlore and polymer matrix.

The asymmetry ratio $\left(A_{21}\right)$, i.e. the intensity ratio of the integrated MDT to EDT peaks, is quite informative form comparing the local environment of $\mathrm{Eu}^{3+}$ ions in different systems. By close check of the emission spectra shown in Fig. $3 b$, the asymmetry ratio of the fibers was found to be lower compared to that of the films. This suggests that the local environment around $\mathrm{Eu}^{3+}$ ions in the fibers is less distorted and more symmetric compared to that in the films. This conclusion is also supported by the observed higher intensity of the other EDT peak corresponding to ${ }^{5} D_{0} \rightarrow{ }^{7} F_{4}$ transition $(\sim 712 \mathrm{~nm})$ from the LHOE-PVDF films compared to the fibers.

The number of Stark splitting of the MDT and EDT peaks is a critical parameter to evaluate point group symmetry around $\mathrm{Eu}^{3+}$ ions. We have recorded emission spectra $\left(\lambda_{\mathrm{ex}}=254 \mathrm{~nm}\right)$ selectively corresponding to the MDT $\left({ }^{5} D_{0} \rightarrow{ }^{7} F_{1}\right)$ and EDTs $\left({ }^{5} D_{0} \rightarrow{ }^{7} F_{2}\right.$ and $\left.{ }^{5} D_{0} \rightarrow{ }^{7} F_{4}\right)$ from the LHOE-PVDF films and fibers (Fig. 4). The increment used is $0.1 \mathrm{~nm}$ per second and the band pass were fixed at $10 \mathrm{~nm}$.

The deduced numbers of Stark components are tabulated in Table 1. The extent of splitting is higher in the fibers compared to the films. As there are five Stark components from the ${ }^{5} \mathrm{D}_{0} \rightarrow{ }^{7} \mathrm{~F}_{4}$ transition of the fibers and three from that of the films, we have concluded that the point group of $\mathrm{Eu}^{3+}$ ions in the LHOE-PVDF fibers and films is $C_{3 v}$ and $D_{2 d}$, respectively.

But we would like to clearly mention here that in relation to Stark component determination; measurement at low temperature and higher spectral resolution would be necessary for a more reliable comparison.

Also, higher emission output was observed from the LHOE-PVDF films as compared to the fibers. This may be related to a well-defined morphology of the films compared to the fibers. As discussed earlier, the fibers have a large degree of entanglement and their surface is nonhomogenous. Both factors may cause substantial scattering of excitation/emission light compared to the films.

We also want to emphasize here that photophysical properties of pristine LHOE powder NPs published before $[5,7,21]$ looks very similar to that of film in terms of emission bandwidth and asymmetry ratio. Only difference is increase in stark component of MDT to 3 in film compared 2 in pristine powder. But the spectra changes substantially when LHOE powder incorporated in PVDF fiber with reduced asymmetry ratio, reduced intensity and slightly more stark component. The increase in stark component is attributed large local field induced in fiber compared to film and powder (Table 1). The reduced asymmetry is because of more homogenous local site offered to $\mathrm{Eu}^{3+}$ ion in fiber compared to pristine powder. The relative excitation and emission intensities of the nanocomposite film 

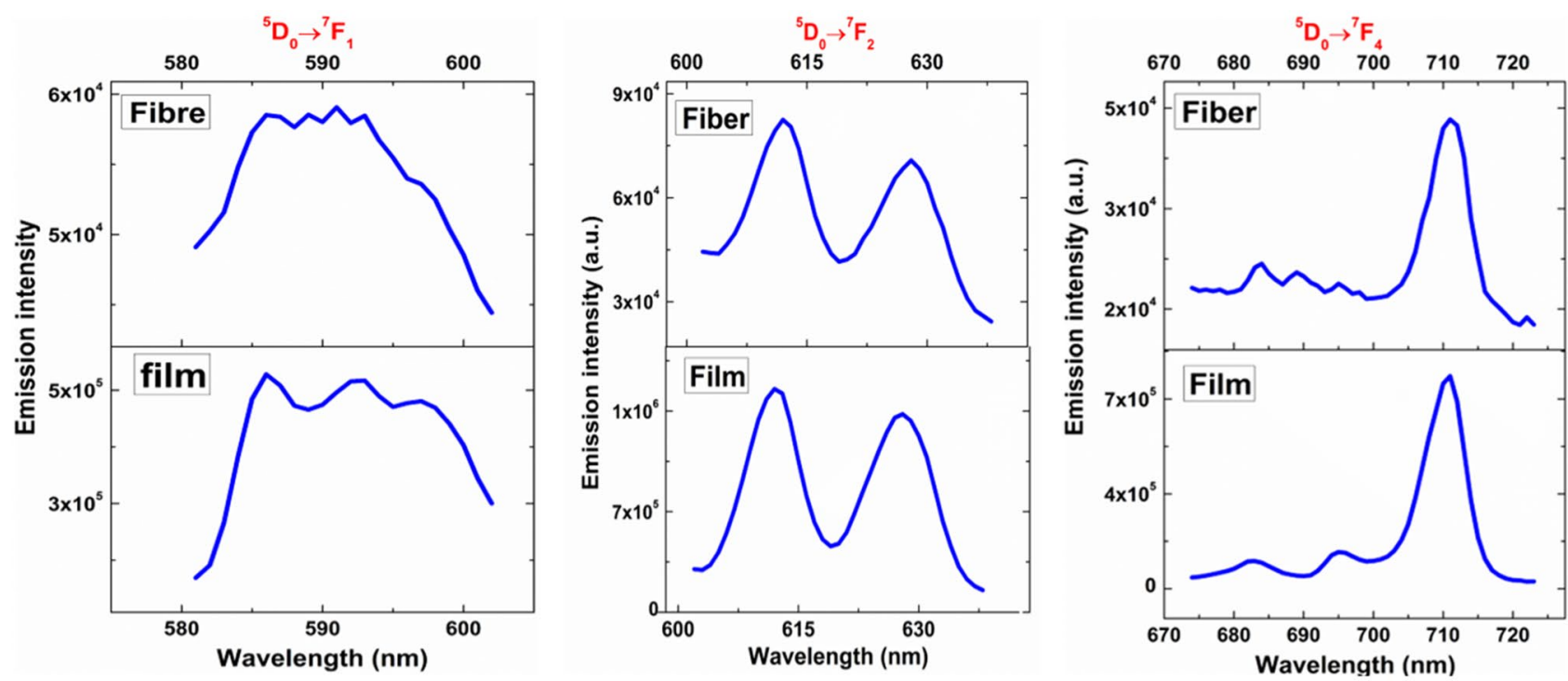

Fig. 4 Emission spectra $\left(\lambda_{\text {ex }}-254 \mathrm{~nm}\right.$ ) of the LHOE-PVDF fibers (top) and films (bottom) by selective zoning the spectral range for MDT and EDT corresponding to the ${ }^{5} \mathrm{D}_{0} \rightarrow{ }^{7} \mathrm{~F}_{1}, \mathrm{D}_{0} \rightarrow{ }^{7} \mathrm{~F}_{2}$, and ${ }^{5} \mathrm{D}_{0} \rightarrow{ }^{7} \mathrm{~F}_{4}$ transitions

Table 1 The number of Stark components from the emission spectra corresponding to the ${ }^{5} \mathrm{D}_{0} \rightarrow{ }^{7} \mathrm{~F}_{1},{ }^{5} \mathrm{D}_{0} \rightarrow{ }^{7} \mathrm{~F}_{2}$, and ${ }^{5} \mathrm{D}_{0} \rightarrow{ }^{7} \mathrm{~F}_{4}$ bands of the LHOE-PVDF fibers and films

\begin{tabular}{llll}
\hline Transitions of $\mathrm{Eu}^{3+}$ & \multicolumn{2}{l}{ Number of Stark components } \\
\cline { 2 - 4 } ions & Films & Fibers & $\begin{array}{l}\text { LHOE } \\
\text { powder } \\
\text { [7] }\end{array}$ \\
\hline${ }^{5} \mathrm{D}_{0} \rightarrow{ }^{7} \mathrm{~F}_{1}$ & 3 & 5 & 2 \\
${ }^{5} \mathrm{D}_{0} \rightarrow{ }^{7} \mathrm{~F}_{2}$ & 2 & 2 & 2 \\
${ }^{5} \mathrm{D}_{0} \rightarrow{ }^{7} \mathrm{~F}_{4}$ & 3 & 5 & 3 \\
\hline
\end{tabular}

as well as fiber are definitely going to be lower than those of the LHOE NPs because of the presence of a reduced number of $\mathrm{Eu}^{3+}$ ions per unit volume.

\subsection{Color coordinates and luminescence lifetime of the LHOE-PVDF films and fibers}

The optical performance of the synthesized LHOE-PVDF films and fibers was also tested for their color coordinate values and excited-state lifetimes. The digital image of the LHOE-PVDF films and fibers under 254 nm UV lamp (Fig. 5)

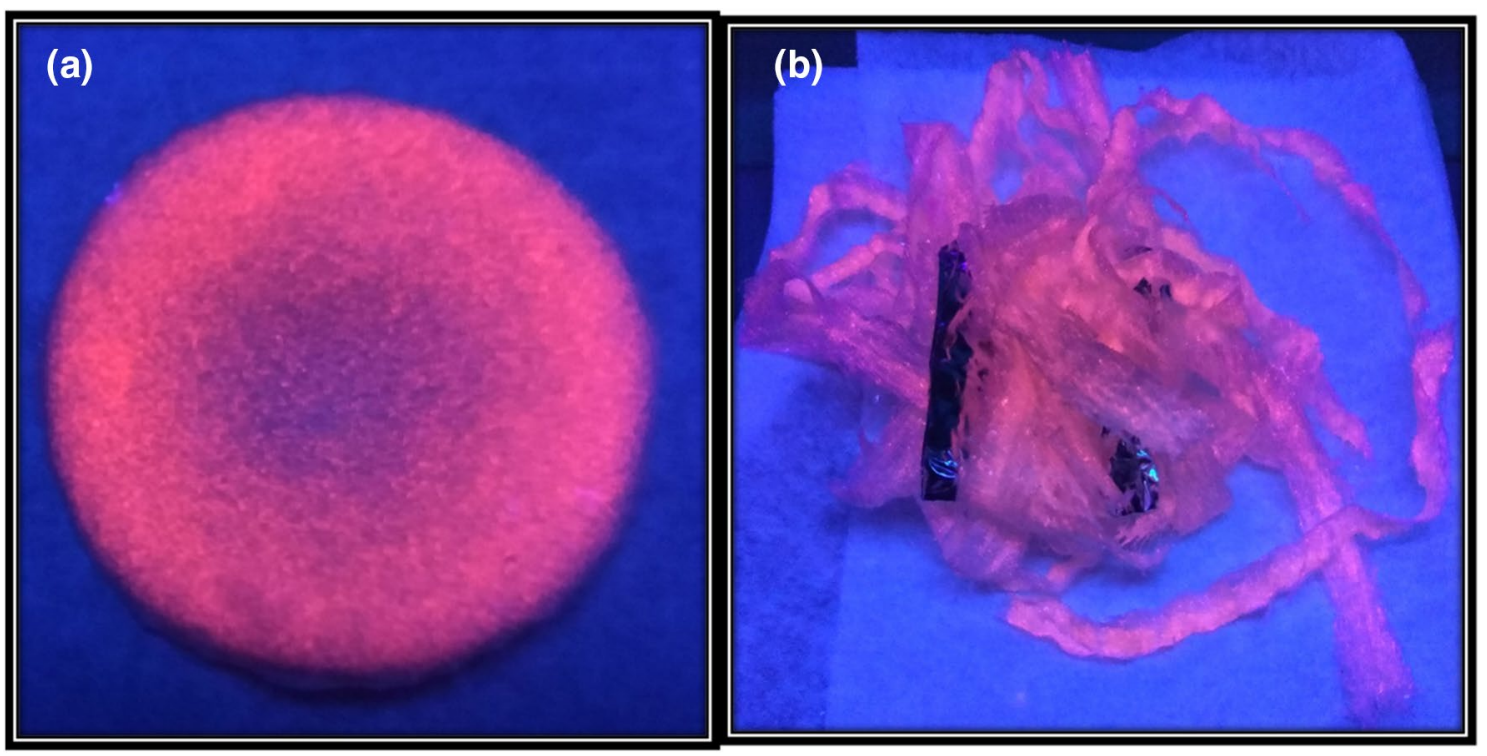

Fig. 5 Digital images of the LHOE-PVDF $\mathbf{a}$ films and $\mathbf{b}$ fibers under $254 \mathrm{~nm}$ excitation 
displays bright red emission due to the intense ${ }^{5} \mathrm{D}_{0}-{ }^{-} \mathrm{F}_{2}$ transition of $\mathrm{Eu}^{3+}$ ions.

The color coordinates evaluated for the LHOE-PVDF films and fibers are shown in the chromaticity diagram (Fig. 6a). The CIE values of the films and fibers are (0.635, $0.352)$ and $(548,0.435)$, respectively, which suggested that red color is shifted towards the yellow-orange domain from the fibers. The observed color difference is from morphological changes rather than PVDF polymer effect.

Exited state lifetime of luminescent materials is another critical quantity for applications related to imaging and optoelectronics. The lifetime decay profile is recorded with $254 \mathrm{~nm}$ excitation and $615 \mathrm{~nm}$ emission for both LHOE-PVDF film and fiber and is shown respectively in Fig. $6 \mathrm{~b}$ and $6 \mathrm{c}$. The decay profiles of the LHOEPVDF films and fibers (Fig. 6b, c, respectively) can be fitted with monoexponential a equation, which suggests that $\mathrm{Eu}^{3+}$ ions are homogeneously distributed in both the films and fibers. The excited-state lifetime values obtained after fitting are $1.94 \mathrm{~ms}$ for the films and $1.61 \mathrm{~ms}$ for the fibers. The higher lifetime values for the films is attributed to lower defect density, smoother surface, and less light scattering compared to the fibers.

Figure 7 gives the summary of entire work wherein MSS LHOE nanoparticles and PVDF polymer solution is drop casted and Forcespun to produce nanocomposite film and fiber which on UV irradiation gives red emission.
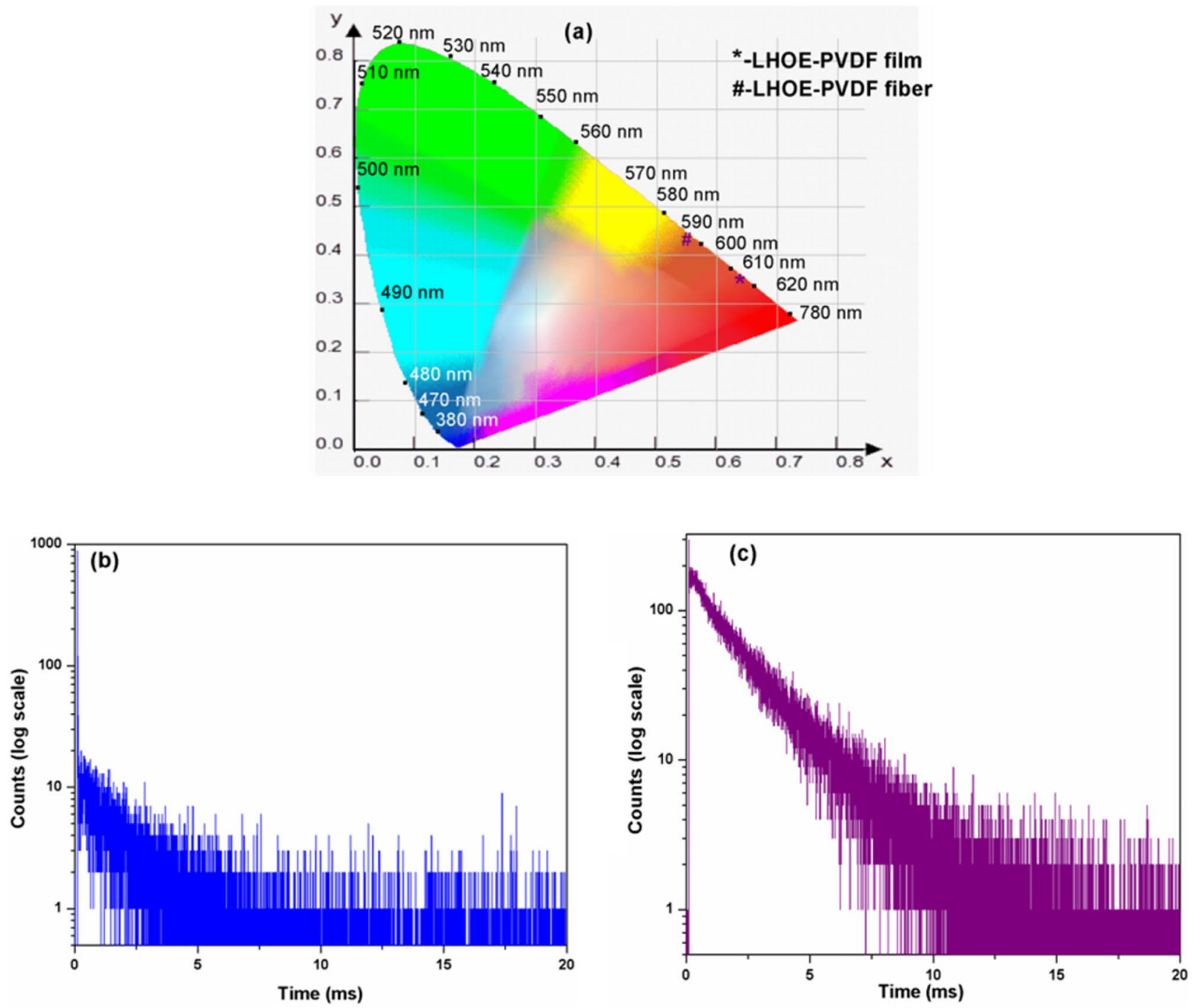

Fig. 6 a CIE index diagram and luminescence lifetime profiles of the LHOE-PVDF $\mathbf{b}$ fibers and $\mathbf{c}$ films under $\lambda_{\mathrm{ex}}=254 \mathrm{~nm}$ and $\lambda_{\mathrm{em}}=615 \mathrm{~nm}$ 


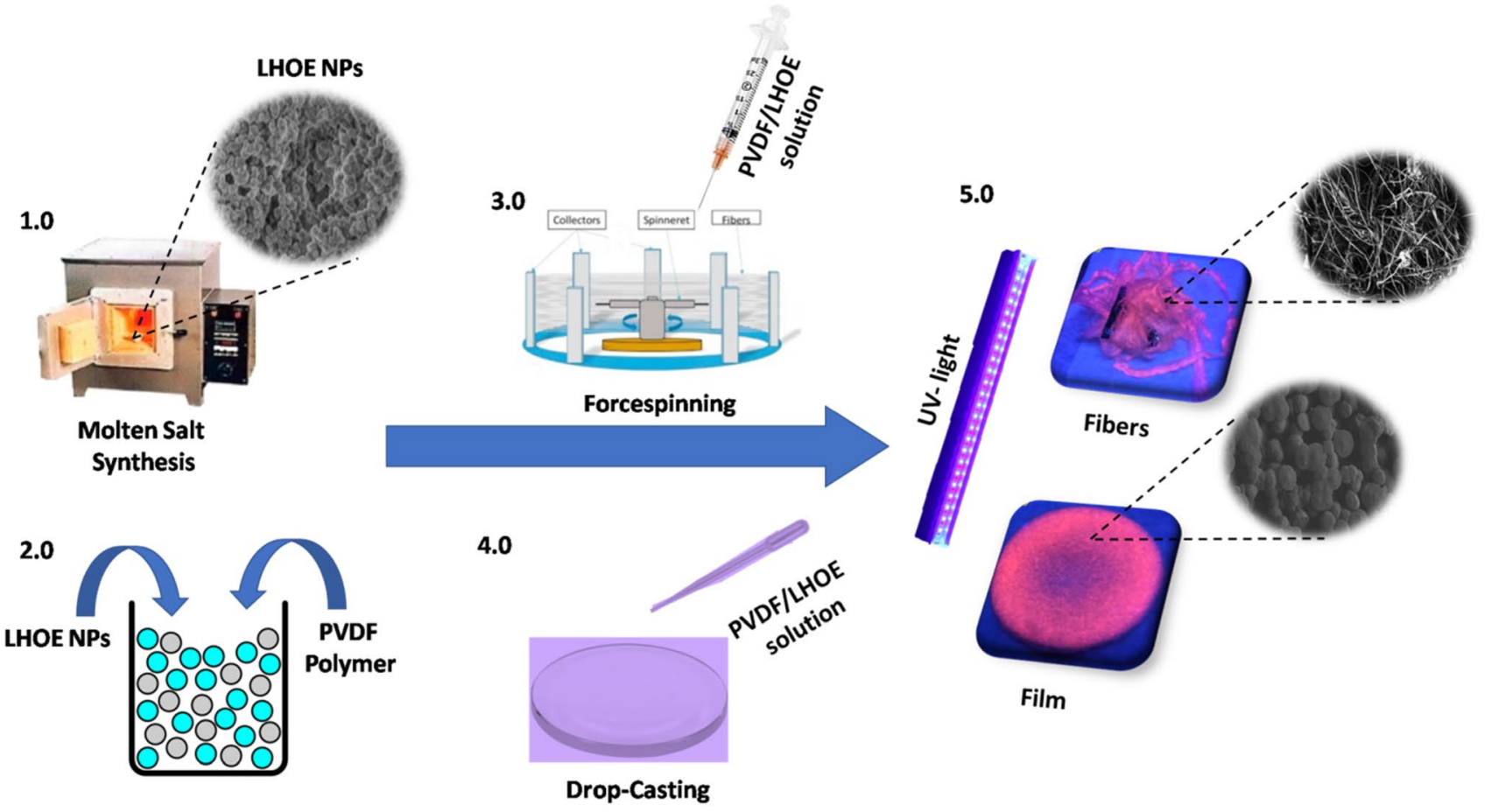

Fig. 7 Schematic showing the synthesis of LHOE-PVDF nanocomposite film and fiber and their downconversion luminescence on UV irradiation. Number in the figure indicates steps

Table 2 Calculated J-O parameters and radiative properties of the LHOE-PVDF fibers and films

\begin{tabular}{lcc}
\hline J-O parameters & Films & Fibers \\
\hline$\tau(\mathrm{ms})$ & 1.61 & 1.94 \\
$A_{\mathrm{RAD}}\left(\mathrm{s}^{-1}\right)$ & 309 & 180 \\
$A_{\mathrm{NRAD}}\left(\mathrm{s}^{-1}\right)$ & 206 & 440 \\
$\eta(\%)$ & 60 & 29 \\
$I_{02} / I_{01}$ & 3.59 & 1.71 \\
$\Omega_{2}\left({ }^{*} 10^{-20} \mathrm{~cm}^{2}\right)$ & 2.15 & 1.02 \\
$\Omega_{4}\left({ }^{*} 10^{-20} \mathrm{~cm}^{2}\right)$ & 1.32 & 1.17 \\
$\beta_{1}$ & 16.2 & 27.7 \\
$\beta_{2}$ & 58.1 & 47.4 \\
$\beta_{4}$ & 16.4 & 24.9 \\
\hline
\end{tabular}

\subsection{Judd-Ofelt analysis of the LHOE-PVDF films and fibers}

Based on Judd-Ofelt analysis and with the help of corrected emission spectra, one can determine various photophysical properties of luminescent materials, such as radiative and non-radiative transition rates/lifetimes, internal quantum efficiency, branching ratios, Judd-Ofelt parameters, etc. $[9,15,48]$. These values were also determined for the LHOE-PVDF fibers and films (Table 2). From this table, it was clearly seen that the non-radiative transition probability is higher in the fiber than the films. This is again attributed to higher defect density and high degree of entanglement plus non-smooth surface of the fibers compared to the films. Defects are known to provide alternative pathways for non-radiative relaxation. The fiber entanglement presents a non-uniform surface, which may cause substantial light scattering. This is reflected in the PL quantum efficiency, which is calculated using radiative and non-radiative lifetimes (inverse of radiative and non-radiative transition rates). The luminescence quantum efficiency of the films (60\%) is much higher than that of fibers (29\%) even though there is not much difference of their excited state lifetime values $(1.94 \mathrm{~ms}$ for the films and 1.61 for the fibers). This is mainly due to large non-radiative channels existing in the fibers compared to the films facilitated by intrinsic defects and non-uniform surface texture.

It is well known that the short-range J-O parameter $\Omega_{2}$ defines the degree of covalency of Eu-O bond and gives information about local structure around the vicinity of the $\mathrm{Eu}^{3+}$ ions whereas the long-range JO parameter $\Omega_{4}$ characterizes the bulk properties such as viscosity and rigidity of the inorganic host matrix where $\mathrm{Eu}^{3+}$ ions are doped into. Higher $\Omega_{2}$ values suggest a higher degree of covalency and more polarizable environments around $\mathrm{Eu}^{3+}$ ions. $\Omega_{2}$ of the film (2.15) is almost double than that of fiber (1.01), suggesting that the $\mathrm{Eu}-\mathrm{O}$ bond is more polarizable and possesses higher covalency in the LHOE-PVDF films compared to the fibers. Also, $\Omega_{2} / \Omega_{4}$ ratio indicates asymmetry of $\mathrm{Eu}^{3+}$ environment, which is $>1$ for the films and $<1$ for 
the fibers. This is entirely consistent with the $A_{21}$ value previously calculated from the emission spectra (Fig. 3b). This suggests that $\mathrm{Eu}^{3+}$ ions have a highly asymmetric structure in the film compared to the fibers. This aspect is also clearly collaborating in branching ratio values $\beta_{n}$ ( $n=1,2$, and 4 ). Higher $\beta_{1}$ and lower $\beta_{2}$ values in the fibers compared to the films indicate a lower red color purity in the fibers due to yellow-orange color compared to the films, consistent with the CIE values shown in Fig. 6a. Infact in powder sample with polymer also had similar trends where in $\Omega 2$ value was found to be more than $\Omega 4$ at all conditions of $\mathrm{pH}$, time and temperature. Branching ratio is predominantly high for $\beta_{2}$ compared to $\beta_{1}$. This suggests all the samples have larger fraction of red emitting photons. The only value which will be different is $\Omega_{2} / \Omega_{4}$ ratio.

\section{Conclusions}

LHOE-PVDF nanocomposite films and fibers were fabricated to explore their potential use in the areas of luminescence. Based on photoluminescence studies, it was inferred that incorporating LHOE NPs into PVDF matrix with different geometries (films and fibers) significantly distorts the local structure around $\mathrm{Eu}^{3+}$ ions due to lattice strain and combined local field effect from the inorganic host and polymer matrix resulting in an enhanced EDT compared to MDT. Based on Stark splitting, we found that point group symmetry around $\mathrm{Eu}^{3+}$ ions in the films is $D_{2 \mathrm{~d}}$ and $C_{3 v}$ for the fibers. Comparison of asymmetry ratio and Judd-Ofelt parameters (such as $\Omega_{2} / \Omega_{4}$ ratio) confirmed that $\mathrm{Eu}^{3+}$ ions in the films have a higher asymmetric environment compared to the fibers. This is further reflected in the higher excited-state lifetime red color purity, and color coordinate values of the films. PL lifetime values are of $1.93 \mathrm{~ms}$ for the films and $1.61 \mathrm{~ms}$ for the fibers. $\Omega_{2}$ value of the films (2.15) is higher than that of the fibers (1.05), suggesting that the chemical environment of $\mathrm{Eu}^{3+}$ ions in the films is more polarizable compared to the fibers and a higher degree of covalency of the Eu-O bond within the films. The luminescent quantum efficiency of the films is higher than the fibers, which is attributed to a large number of non-radiative channels in the fibers. Based on these studies, we conclude that the LHOE-PVDF nanocomposite films possess better optical properties and can be utilized in futuristic display devices. This work not only highlights the importance of phosphor films and fibers but also opens up new opportunities for optical scientists to make their nanomaterials commercial for societal applications.

Acknowledgement YM thanks the financial support by the National Science Foundation under CHE (award \#1710160) and the IIT startup funds. SKG thanks the United States-India Education Foundation
(USIEF, India) and the Institute of International Education (IIE, USA) for his Fulbright Nehru Postdoctoral Fellowship (Award\# 2268/ FNPDR/2017).

\section{Compliance with ethical standards}

Conflict of interest The authors declare that they have no conflict of interest.

\section{References}

1. Sun S-K, Wang H-F, Yan X-P (2018) Engineering persistent luminescence nanoparticles for biological applications: from biosensing/bioimaging to theranostics. Acc Chem Res 51:1131-1143

2. Liu J, Lécuyer T, Seguin J, Mignet N, Scherman D, Viana B, Richard C (2019) Imaging and therapeutic applications of persistent luminescence nanomaterials. Adv Drug Deliv Rev 138:193-210

3. Valenti G, Rampazzo E, Kesarkar S, Genovese D, Fiorani A, Zanut A, Palomba F, Marcaccio M, Paolucci F, Prodi L (2018) Electrogenerated chemiluminescence from metal complexes-based nanoparticles for highly sensitive sensors applications. Coord Chem Rev 367:65-81

4. Guzman M, Flores B, Malet L, Godet S (2018) Synthesis and characterization of zinc oxide nanoparticles for application in the detection of fingerprints. Mater Sci Forum 916:232-236

5. Gupta SK, Zuniga JP, Abdou M, Thomas MP, Goonatilleke MDA, Guiton BS, Mao Y (2020) Lanthanide-doped lanthanum hafnate nanoparticles as multicolor phosphors for warm white lighting and scintillators. Chem Eng J 379:122314

6. Gupta SK, Abdou M, Zuniga JP, Puretzky AA, Mao Y (2019) Samarium-activated $\mathrm{La}_{2} \mathrm{Hf}_{2} \mathrm{O}_{7}$ nanoparticles as multifunctional phosphors. ACS Omega 4:17956-17966

7. Gupta SK, Zuniga JP, Ghosh PS, Abdou M, Mao Y (2018) Correlating Structure and luminescence properties of undoped and $\mathrm{Eu}^{3+}$-doped $\mathrm{La}_{2} \mathrm{Hf}_{2} \mathrm{O}_{7}$ nanoparticles prepared with different coprecipitating ph values through experimental and theoretical studies. Inorg Chem 57:11815-11830

8. Hernandez C, Gupta SK, Zuniga JP, Vidal J, Galvan R, Martinez M, Guzman H, Chavez L, Mao Y, Lozano K (2019) Performance evaluation of $\mathrm{Ce}^{3+}$ doped flexible PVDF fibers for efficient optical pressure sensors. Sens Actuators, A 298:111595

9. Gupta SK, Mohapatra M, Natarajan V, Godbole SV (2012) Sitespecific luminescence of $\mathrm{Eu}^{3+}$ in gel-combustion-derived strontium zirconate perovskite nanophosphors. J Mater Sci 47:3504-3515

10. Zuniga JP, Abdou M, Gupta SK, Mao Y (2018) Molten-salt synthesis of complex metal oxide nanoparticles. J Vis Exp 140:e58482

11. Chander H (2006) A review on synthesis of nanophosphorsfuture luminescent materials. In: Proceedings of ASID'06 pp 8-12

12. Penilla Garcia MA, Gupta SK, Mao Y (2020) Effects of moltensalt processing parameters on the structural and optical properties of preformed $\mathrm{La}_{2} \mathrm{Zr}_{2} \mathrm{O}_{7}: \mathrm{Eu}^{3+}$ nanoparticles. Ceram Int 46:1352-1361

13. Shi J, Ge W, Xu M, Zhu J (2019) $\mathrm{Bi}_{2} \mathrm{Ti}_{2} \mathrm{O}_{7}$ nanoparticles: An oxide based upconversion luminescence host by a simple sol-gel route. J Lumin 213:15-18

14. Jena P, Gupta SK, Natarajan V, Sahu M, Satyanarayana N, Venkateswarlu M (2015) Structural characterization and photoluminescence properties of sol-gel derived nanocrystalline BaMoO4:Dy3+. J Lumin 158:203-210 
15. Gupta SK, Sahu M, Krishnan K, Saxena MK, Natarajan V, Godbole SV (2013) Bluish white emitting $\mathrm{Sr}_{2} \mathrm{CeO}_{4}$ and red emitting $\mathrm{Sr}_{2} \mathrm{CeO}_{4}: \mathrm{Eu}^{3+}$ nanoparticles: optimization of synthesis parameters, characterization, energy transfer and photoluminescence. J Materials Chem C 1:7054-7063

16. Zuniga JP, Gupta SK, Abdou M, De Santiago HA, Puretzky AA, Thomas MP, Guiton BS, Liu J, Mao Y (2019) Size, structure, and luminescence of $\mathrm{Nd} 2 \mathrm{Zr} 2 \mathrm{O} 7$ nanoparticles by molten salt synthesis. J Mater Sci 54:12411-12423

17. Rafique R, Baek SH, Phan LMT, Chang S-J, Gul AR, Park TJ (2019) A facile hydrothermal synthesis of highly luminescent $\mathrm{NaYF}_{4}: \mathrm{Yb}^{3+}$ / $\mathrm{Er}^{3+}$ upconversion nanoparticles and their biomonitoring capability. Mater Sci Eng, C 99:1067-1074

18. Gupta SK, Gupta R, Natarajan V, Godbole SV (2014) Warm white light emitting $\mathrm{ThO}_{2}: \mathrm{Sm}^{3+}$ nanorods: cationic surfactant assisted reverse micellar synthesis and photoluminescence properties. Mater Res Bull 49:297-301

19. Liu D, Tse K, Robertson J (2007) Electronic structure and defects of high dielectric constant gate oxide $\mathrm{La}_{2} \mathrm{Hf}_{2} \mathrm{O}_{7}$. Appl Phys Lett 90:062901

20. Hansel RA, Desai SK, Allison SW, Heyes AL, Walker DG (2010) Emission lifetimes of europium-doped pyrochlores for phosphor thermometry. J Appl Phys 107:016101

21. Zuniga JP, Gupta SK, Abdou M, Mao Y (2018) Effect of molten salt synthesis processing duration on the photo- and radioluminescence of UV-, visible-, and X-ray-excitable $\mathrm{La}_{2} \mathrm{Hf}_{2} \mathrm{O}_{7}: \mathrm{Eu}^{3+}$ nanoparticles. ACS Omega 3:7757-7770

22. Sun H, Li Z, Kong L, Wang B, Zhang C, Yuan Q, Huang S, Liu Y, $\mathrm{Li} \mathrm{L} \mathrm{(2018)} \mathrm{Enhancing} \mathrm{the} \mathrm{stability} \mathrm{of} \mathrm{CsPbBr}_{3}$ nanocrystals by sequential surface adsorption of ${ }^{\mathrm{S} 2}-$ and metal ions. Chem Commun 54:9345-9348

23. Bispo-Jr AG, Oliveira NA, Cardoso CX, Lima SAM, Job AE, Osorio-Román IO, Danna CS, Pires AM (2018) Red-light-emitting polymer composite based on PVDF membranes and Europium phosphor using Buriti Oil as plasticizer. Mater Chem Phys 217:160-167

24. Jia J, Li D-X, Jia H-S, Zhang A-Q, Tao P, Liu X-G (2017) Effect of composition and package structure of bi-color flexible remote phosphor film on the properties of remote white LEDs. Opt Mater 72:602-611

25. Jaffar BM, Swart HC, Seed Ahmed HAA, Yousif A, Kroon RE (2019) Stability of Bi doped $\mathrm{La}_{2} \mathrm{O}_{3}$ powder phosphor and PMMA composites. J Phys Chem Solids 131:156-163

26. Khursheed S, Biswas P, Singh VK, Kumar V, Swart HC, Sharma $\mathrm{J}$ (2019) Synthesis and optical studies of $\mathrm{KCaVO}_{4}: \mathrm{Sm}^{3+} / \mathrm{PMMA}$ nanocomposites. Vacuum 159:414-422

27. Prakash J, Kumar V, Erasmus LJB, Duvenhage MM, Sathiyan G, Bellucci S, Sun S, Swart HC (2018) Phosphor polymer nanocomposite: $\mathrm{ZnO}: \mathrm{Tb}^{3+}$ embedded polystyrene nanocomposite thin films for solid-state lighting applications. ACS Appl Nano Materials 1:977-988

28. Gupta SK, Zuniga JP, Abdou M, Ghosh PS, Mao Y (2020) Optical properties of undoped, $\mathrm{Eu}^{3+}$ doped and $\mathrm{Li}^{+}$co-doped $\mathrm{Y}_{2} \mathrm{Hf}_{2} \mathrm{O}_{7}$ nanoparticles and polymer nanocomposite films. Inorg Chem Front 7:505-518

29. Jia N, He Q, Sun J, Xia G, Song R (2017) Crystallization behavior and electroactive properties of PVDF, P(VDF-TrFE) and their blend films. Polym Test 57:302-306

30. Khursheed S, Kumar V, Singh VK, Sharma J, Swart HC (2018) Optical properties of $\mathrm{Sr}_{3} \mathrm{~B}_{2} \mathrm{O}_{6}$ :Dy ${ }^{3+} /$ PMMA polymer nanocomposites. Phys B 535:184-188

31. McSherry M, Fitzpatrick C, Lewis E (2005) Review of luminescent based fibre optic temperature sensors. Sens Rev 25:56-62
32. Shingange $\mathrm{K}$, Swart $\mathrm{HC}$, Mhlongo $\mathrm{GH}$ (2020) $\mathrm{LaBO}_{3}(\mathrm{~B}=\mathrm{Fe}, \mathrm{Co})$ nanofibers and their structural, luminescence and gas sensing characteristics. Phys B: Condens Matter 578:411883

33. Antoniadou M, Pilch-Wrobel A, Riziotis C, Bednarkiewicz A, Tanasă E, Krasia-Christoforou T (2019) Fluorescent electrospun PMMA microfiber mats with embedded $\mathrm{NaYF}_{4}: \mathrm{Yb} /$ Er upconverting nanoparticles. Methods Appl Fluoresc 7:034002

34. Kunti AK, Sharma SK, Choudhary RJ, Swart HC (2019) Structural and luminescence properties of laser assisted $\mathrm{Eu}^{3+}$ doped $\mathrm{BaZrO}_{3}$ thin films. J Alloy Compd 801:99-111

35. Zhang L, Lyu S, Chen Z, Wang S (2018) Fabrication flexible and luminescent nanofibrillated cellulose films with modified $\mathrm{SrAl}_{2} \mathrm{O}_{4}$ :Eu. Dy phosphors via nanoscale silica and aminosilane, Nanomaterials 8:352

36. Ku AY, Heward WJ, Mani V (2006) Templated synthesis of nanostructured europium-doped yttrium oxide thin films. J Mater Sci 41:3017-3025

37. Shimizu $Y$, Ueda $K$ (2016) Determination of $4 f$ energy levels for trivalent lanthanide ions in $\mathrm{YAlO}_{3}$ by $\mathrm{X}$-ray photoelectron spectroscopy. Thin Solid Films 614:69-72

38. Lozano K, Sarkar K (2009) Methods and apparatuses for making superfine fibers, Google Patents, 2009.

39. Sarkar K, Gomez C, Zambrano S, Ramirez M, de Hoyos E, Vasquez $\mathrm{H}$, Lozano K (2010) Electrospinning to forcespinning ${ }^{\mathrm{TM}}$. Mater Today 13:12-14

40. Akia M, Salinas N, Luna S, Medina E, Valdez A, Lopez J, Ayala J, Alcoutlabi M, Lozano K (2019) In situ synthesis of $\mathrm{Fe}_{3} \mathrm{O}_{4}$-reinforced carbon fiber composites as anodes in lithiumion batteries. J Mater Sci 54:13479-13490

41. Liu S, Reneker DH (2019) Droplet-jet shape parameters predict electrospun polymer nanofiber diameter. Polymer 168:155-158

42. Flores D, Villarreal J, Lopez J, Alcoutlabi M (2018) Production of carbon fibers through Forcespinning ${ }^{\circledR}$ for use as anode materials in sodium ion batteries. Mater Sci Eng, B 236:70-75

43. Maystre D (2012) Theory of Wood's anomalies. Plasmonics. Springer, Berlin, pp 39-83

44. Jameson DM (2014) Introduction to fluorescence. CRC Press, Boca Raton

45. Zuniga JP, Gupta SK, Pokhrel M, Mao Y (2018) Exploring the optical properties of La2Hf2O7:Pr3+ nanoparticles under UV and $X$-ray excitation for potential lighting and scintillating applications. New J Chem 42:9381-9392

46. Gupta SK, Zuniga JP, Abdou M, Mao Y (2018) Thermal annealing effects on $\mathrm{La}_{2} \mathrm{Hf}_{2} \mathrm{O}_{7}: \mathrm{Eu}^{3+}$ nanoparticles: a curious case study of structural evolution and site-specific photo- and radio-luminescence. Inorg Chem Front 5:2508-2521

47. Abdou M, Gupta SK, Zuniga JP, Mao Y (2018) On structure and phase transformation of uranium doped $\mathrm{La}_{2} \mathrm{Hf}_{2} \mathrm{O}_{7}$ nanoparticles as an efficient nuclear waste host. Mater Chem Front 2:2201-2211

48. Keskar M, Gupta SK, Phatak R, Kannan S, Natarajan V (2015) Optical properties of $\mathrm{Eu}^{3+}$ activated thorium molybdate and thorium tungstate: structure, local symmetry and photophysical properties. J Photochem Photobiol, A 311:59-67

Publisher's Note Springer Nature remains neutral with regard to jurisdictional claims in published maps and institutional affiliations. 